\title{
Quantum Entanglement and Controlled Logical Gates Using Coupled SQUID Flux Qubits
}

\author{
Zhongyuan Zhou, Shih-I Chu, and Siyuan Han
}

\begin{abstract}
We present an approach to realize universal two-bit quantum gates using two SQUID flux qubits. In this approach the basic unit consists of two inductively coupled SQUIDs with realistic device parameters. Quantum logical gates are implemented by applying resonant microwave pulse to the qubits. This procedure is demonstrated by realizing a controlled-NOT (CNOT) gate and the maximally entangled states of the coupled qubits through highly accurate numerical solution of the time-dependent Schrödinger equation of the system. This coupling scheme is simple and can be readily extended to many-qubit circuits required for scalable quantum information processing.
\end{abstract}

Index Terms-Coupled SQUID flux qubits, quantum computing, quantum entanglement, two-bit gates.

\section{INTRODUCTION}

$\mathbf{S}$ OLID-STATE qubits are of particular interest because they have the advantages of large-scale integration, flexibility in design, and easy connection to conventional electronic circuits. Of the solid-state qubits, superconducting qubits based on Josephson junctions (JJ) have recently attracted more attention [1]. A variety of single qubits, such as JJ charge qubits [2], [3], JJ phase qubits [4]-[6], and superconducting quantum interference device (SQUID) flux qubits [7]-[9], have been implemented by using the coherence of Cooper-pair tunneling in the superconducting state. However, a practical quantum computer will be comprised of a set of coupled qubits [10]. Progress has been made toward this goal. Temporal quantum coherent state and Rabi oscillation of two coupled JJ charge qubits [11], [12] and spectroscopy evidence of entangled state in two-coupled JJ phase qubits [13] have been demonstrated experimentally.

Nevertheless, the charge qubits usually have stronger decoherence than the flux qubits because the charge noise is generally more difficult to suppress than the flux noise [14]. Decoherence, such as dephasing and energy relaxation [14], [15], destroys quantum coherence of qubit states that is critical to quantum computation and communication [1], [10], [16]. Recent experiments showed that the rf-driven superconducting flux qubit yielded a relaxation time of $900 \mathrm{~ns}$ and a dephasing time of $20 \mathrm{~ns}$ and is therefore promising for coupled qubits and quantum computation [9].

Manuscript received October 5, 2004. This work was supported in part by the National Science Foundation (NSF) under Grant EIA-0082499 and NSF ITR program DMR-0325551, and in part by the Air Force Office of Scientific Research (AFOSR) under Grant F49620-01-1-0439 funded by the Defense University Research Initiative on Nanotechnology (DURINT) program and by ARDA.

Z. Zhou and S.-I. Chu are with the Department of Chemistry, University of Kansas, Lawrence, KS 66045 USA (e-mail: zyzhou@ku.edul; sichu@ku.edu).

S. Han is with the Department of Physics and Astronomy, University of Kansas, Lawrence, KS 66045 USA (e-mail: han@ku.edu).

Digital Object Identifier 10.1109/TASC.2005.850074
In this paper we demonstrate the implementation of two-bit gates using two SQUID flux qubits. The basic unit consists of two SQUIDs with realistic device parameters coupled via mutual inductance between them. The universal two-bit quantum gates are implemented by applying resonant microwave pulse to the coupled qubits. The feasibility of this procedure is demonstrated by accomplishing entangled states and controlled-NOT (CNOT) gate of the coupled flux qubits through highly accurate numerical solution of time-dependent Schrödinger equation of the system. The proposed scheme of coupled flux qubits is simple and can be experimentally implemented with the methods already developed for single flux qubits [4]-[9].

\section{ENERGY LEVEL OF THE COUPLED RF SQUID QUBITS}

An rf SQUID consists of a superconducting loop of inductance $L$ interrupted by a Josephson tunnel junction. Applying the resistively shunted junction (RSJ) model [17], the junction is characterized by its critical current $I_{\mathrm{c}}$, shunt capacitance $C$, and shunt resistance $R$. A flux-biased SQUID with total magnetic flux $\Phi$ enclosed in the loop is analogous to a "flux" particle of mass $m=C \Phi_{0}^{2}$ (where $\Phi_{0}=h / 2 e$ is the flux quantum) moving in a potential. The Hamiltonian of an rf SQUID can be written as [18]

$$
h(x)=\frac{p^{2}}{2 m}+u(x),
$$

where, $x=\Phi / \Phi_{0}$ is the canonical coordinate of the "flux" particle, $p=-i \hbar \partial / \partial x$ is the canonical momentum conjugate to $x$, and $u(x)$ is the potential energy given by

$$
u(x)=\frac{m \omega_{L C}^{2}\left(x-x_{e}\right)^{2}}{2}-E_{J} \cos (2 \pi x),
$$

here, $E_{\mathrm{J}}=I_{\mathrm{C}} \Phi_{0} / 2 \pi=m \omega_{\mathrm{LC}}^{2} \beta_{\mathrm{L}} / 4 \pi^{2}$ is the Josephson coupling energy, $\omega_{\mathrm{LC}}=(L C)^{-1 / 2}$ is the characteristic frequency of the SQUID, $\beta_{\mathrm{L}}=2 \pi L I_{\mathrm{c}} / \Phi_{0}$ is the potential shape parameter, and $x_{\mathrm{e}}=\Phi_{\mathrm{e}} / \Phi_{0}$ is the normalized external flux bias.

The eigenenergies and eigenstates of a single rf SQUID qubit are obtained by numerically solving the Schrödinger equation with the Hamiltonian $h(x)$ [18]. The eigenstates of the rf SQUID depend only on two independent parameters: the potential shape parameter $\beta_{\mathrm{L}}$ and the characteristic impedance $Z_{0}=(L / C)^{1 / 2}$, while the energy is scaled to $\hbar \omega_{\mathrm{LC}}$ [18]. The SQUID's energy level structure can be controlled in situ by adjusting $\beta_{\mathrm{L}}$ and $x_{\mathrm{e}}$ via changing the critical current $I_{\mathrm{c}}$ and the external flux bias $\Phi_{\mathrm{e}}$. For example, for a fixed $Z_{0}$ the total number of levels in the left and right wells, $N_{\mathrm{L}}+N_{\mathrm{R}}$ increases with $\beta_{\mathrm{L}}$ while the difference, $N_{\mathrm{L}}-N_{\mathrm{R}}$, increases with $x_{\mathrm{e}}$. A good qubit requires two qubit states well separated in space, 


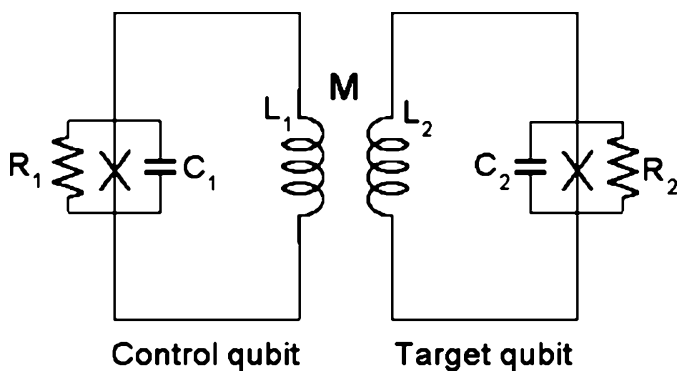

Fig. 1. Circuits for the coupled two-SQUID flux qubits.

i.e., flux. Thus the potential barrier cannot be too small. However, a higher barrier will make the transition matrix elements exponentially smaller and thus decreasing the frequency of the Rabi oscillation. As a compromise we choose SQUIDs with $Z_{0}=50 \Omega$ (i.e., $L=100 \mathrm{pH}$, and $C=40 \mathrm{fF}$ ), $\beta_{\mathrm{L}}=1.2$, and $\omega_{\mathrm{LC}}=5 \times 10^{11} \mathrm{rad} / \mathrm{s}$.

The coupled SQUID qubits consist of two single rf SQUID qubits: a control qubit and a target qubit. The two SQUIDs are coupled inductively by the mutual inductance $M$ as shown in Fig. 1. For simplicity, we assume that the two SQUIDs are identical $\left(C_{1}=C_{2}=C, L_{1}=L_{2}=L\right.$, and $\left.R_{1}=R_{2}=R\right)$. The external magnetic fluxes applied to the two SQUIDs are $\Phi_{\mathrm{e} 1}$ and $\Phi_{\mathrm{e} 2}$, and the total fluxes are $\Phi_{1}$ and $\Phi_{2}$, respectively.

The Hamiltonian of the coupled SQUID qubits is

$$
H\left(x_{1}, x_{2}\right)=h\left(x_{1}\right)+h\left(x_{2}\right)+h_{12}\left(x_{1}, x_{2}\right),
$$

where, $h_{12}\left(x_{1}, x_{2}\right)$ is the interaction between the two SQUIDs, which is given by

$$
\begin{aligned}
h_{12}\left(x_{1}, x_{2}\right) & =\frac{M \Phi_{0}\left(x_{1}-x_{e 1}\right)\left(x_{2}-x_{e 2}\right)}{L^{2}} \\
& =\frac{m \omega_{L C}^{2} k\left(x_{1}-x_{e 1}\right)\left(x_{2}-x_{e 2}\right)}{2} .
\end{aligned}
$$

Here, $x_{\mathrm{i}}=\Phi_{\mathrm{i}} / \Phi_{0}$ and $x_{\mathrm{ei}}=\Phi_{\mathrm{ei}} / \Phi_{0}$ for $i=1$ and 2 , and $k=$ $2 M / L$ is the coupling coefficient. For weakly coupled qubits $k \ll 1$. The potential energy surface of the coupled SQUID qubits for $x_{\mathrm{e} 1}=0.499, x_{\mathrm{e} 2}=0.49895$, and $k=0.001$ is shown in Fig. 2.

The eigenenergies and eigenstates of the coupled qubits are computed by solving the Schrödinger equation with $H\left(x_{1}, x_{2}\right)$ using the two-dimensional Fourier-grid Hamiltonian method [19]. For $k=0$, the two qubits are decoupled and an eigenstate is just a product of an eigenstate of the control qubit and an eigenstate of the target qubit, i.e., $\left.\left|n_{1} n_{2}\right|=\left|n_{1}>\right| n_{2}\right\rangle$, where $\left.\left|n_{1} n_{2}\right|\right)$ denotes the eigenstate of the two-qubit system and $\mid n_{1}>$ and $\mid n_{2}>$ are the eigenstates of the control qubit and target qubit. For example, the four lowest eigenstates are $\mid 00)=|0>| 0>, \mid 01)=|0>| 1>, \mid 10)=|1>| 0>$, and $\mid 11)=|1>| 1>$. For $k \neq 0$, an eigenstate of the coupled qubits is no longer a product of the two single qubits' eigenstates. However for very small $k$ a product of the two single qubits' eigenstates approximates an eigenstate of the two-qubit system well.

In Fig. 3 the energy levels (in units of $\hbar \omega_{\mathrm{LC}}$ ) of the coupled qubits are plotted versus the flux bias of the target qubit $x_{\mathrm{e} 2}$ for $x_{\mathrm{e} 1}=0.499$ and $k=0.001$. In the upper left inset and the upper

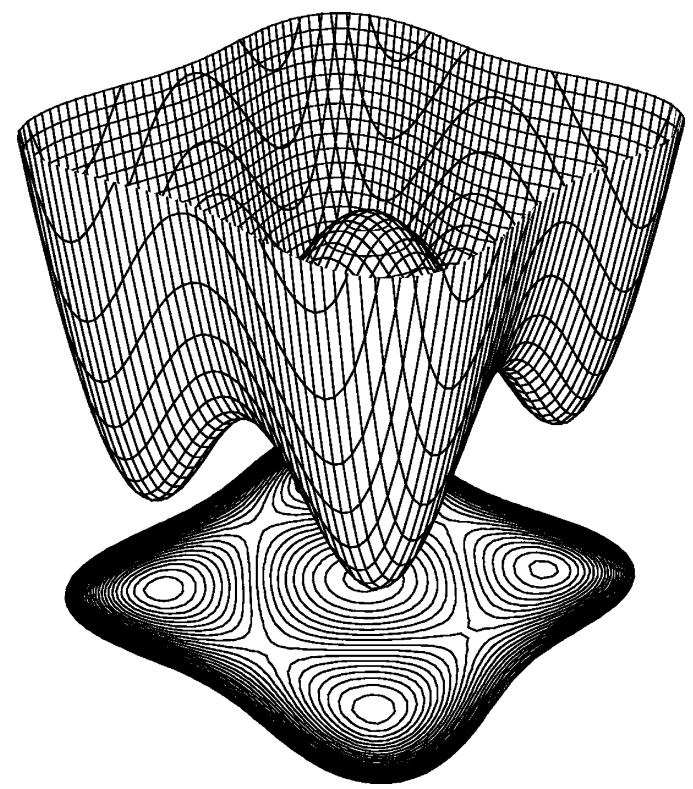

Fig. 2. Potential energy surface of the coupled SQUID qubits with $x_{\mathrm{e} 1}=$ $0.499, x_{\mathrm{e} 2}=0.49895$, and $k=0.001$.

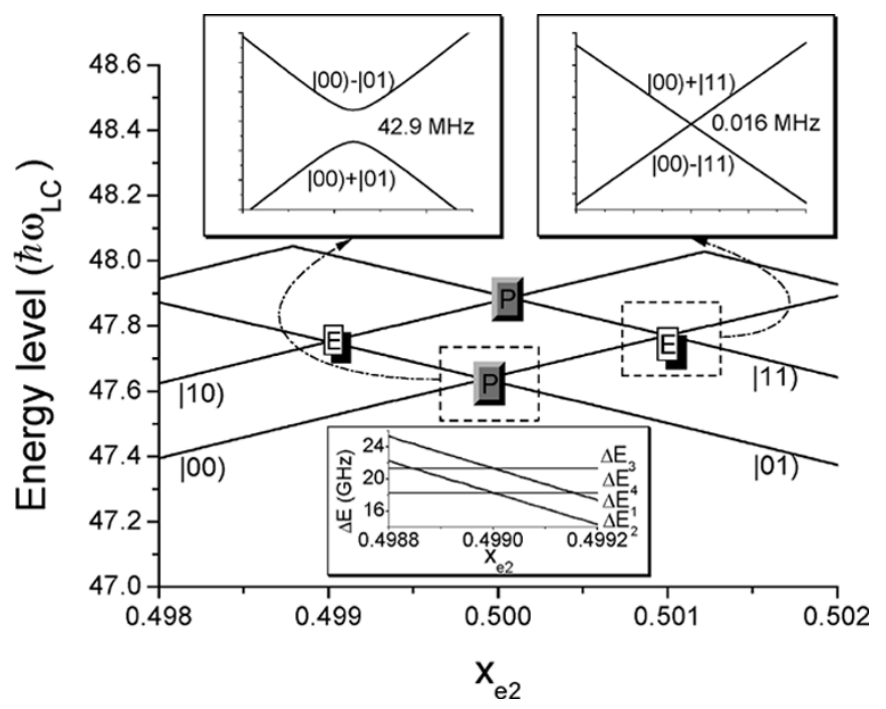

Fig. 3. Energy levels of the coupled qubits versus the bias of the target qubit $x_{\mathrm{e} 2}$ for $x_{\mathrm{e} 1}=0.499$ and $k=0.001$. The two upper insets show a product state and an entangled state at the avoided crossing points. The lower inset displays the energy level spacings $\Delta E_{1}=\mathrm{E}_{\mid 11)}-\mathrm{E}_{\mid 10)}, \Delta E_{2}=\mathrm{E}_{\mid 01)}-\mathrm{E}_{(00)}$, $\Delta E_{3}=\mathrm{E}_{\mid 11)}-\mathrm{E}_{(01)}$, and $\Delta E_{4}=\mathrm{E}_{\mid 10)}-\mathrm{E}_{(00)}$.

right inset we show a product state and an entangled state at the avoided crossing points, respectively ( $\mathrm{P}$ denotes the avoided crossing point with product states and $\mathrm{E}$ the avoided crossing point with entangled states). In the lower inset we display energy level spacings $\Delta E_{1}=\mathrm{E}_{\mid 11)}-\mathrm{E}_{\mid 10)}, \Delta E_{2}=\mathrm{E}_{\mid 01)}-\mathrm{E}_{\mid 00)}$, $\Delta E_{3}=\mathrm{E}_{(11)}-\mathrm{E}_{\mid 01)}$, and $\Delta E_{4}=\mathrm{E}_{\mid 10)}-\mathrm{E}_{\mid 00)}$. Note that $\Delta E_{1}$ and $\Delta E_{2}$ decrease linearly with $x_{\mathrm{e} 2}$ while $\Delta E_{3}$ and $\Delta E_{4}$ do not change at all. At the avoided crossing points, the spacing between the product states in the upper left inset is $42.9 \mathrm{MHz}$ while the spacing between the entangled states in the upper right inset is $0.016 \mathrm{MHz}$, which is much smaller than that of the product states. In the product states the eigenenergy of the state $(00)+\mid 01)$ is lower than that of the state $(00)-\mid 01)$ while in the entangled states the eigenenergy of the state $\mid 00)-\mid 11$ ) 


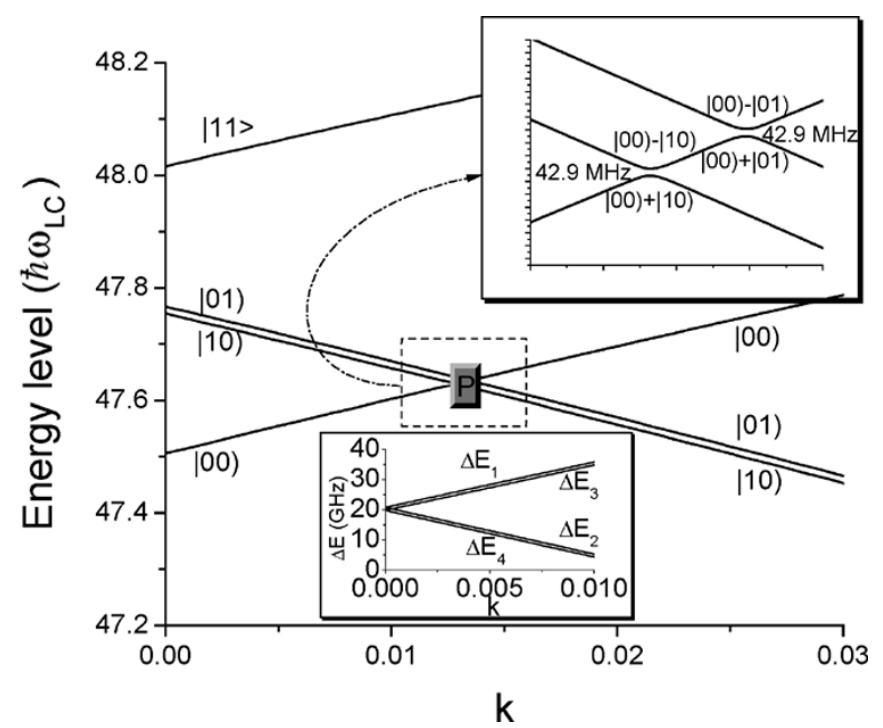

Fig. 4. Energy levels of the coupled qubits versus the coupling coefficient $k$ for $x_{\mathrm{e} 1}=0.499$ and $x_{\mathrm{e} 2}=0.49895$. In the upper inset the product states at the avoided crossing points are shown and in the lower inset the energy level spacings $\Delta E_{1}, \Delta E_{2}, \Delta E_{3}$, and $\Delta E_{4}$ are plotted.

is lower than that of the state $\mid 00)+\mid 11)$. In our method the flux bias points of the quantum logical gates are away from the avoided crossing points.

In Fig. 4 the energy levels of the coupled qubits are plotted as a function of the coupling coefficient $k$ for $x_{\mathrm{e} 1}=0.499$ and $x_{\mathrm{e} 2}=0.49895$. In the upper inset product states at avoided crossing points are shown and in the lower inset the energy level spacings $\Delta E_{1}, \Delta E_{2}, \Delta E_{3}$, and $\Delta E_{4}$ are exhibited. It is clear that $\Delta E_{1}$ and $\Delta E_{3}$ increase linearly with $k$ while the spacings $\Delta E_{2}$ and $\Delta E_{4}$ decrease linearly with $k$. Both the spacings of the product states at the avoided crossing points in the upper inset are $42.9 \mathrm{MHz}$, which is also identical with that in the upper left inset of Fig. 3. Obviously, for two-bit gate operation the coupled qubits should not be in states with $k$ closer and greater than 0.01 . After considering energy level structure and the energy level spacings, we find that one of the proper configurations for implementing CNOT gate is at $x_{\mathrm{e} 1}=0.499, x_{\mathrm{e} 2}=0.49895$, and $k=0.001$. We will use these parameters in the following investigation of two-qubit logical gates.

\section{EnTangled States AND Two-Bit Gates}

To realize two-bit gates of the coupled SQUID flux qubits, a resonant microwave pulse is applied to the target qubit. The interaction between the microwave and the coupled qubits is

$V\left(x_{1}, x_{2}, t\right)=m \omega_{L C}^{2}\left[\left(x_{2}-x_{e 2}\right) x_{\varepsilon}+\frac{x_{\varepsilon}^{2}}{2}+\frac{k\left(x_{1}-x_{e 1}\right) x_{\varepsilon}}{2}\right]$,

where, $x_{\varepsilon}(t)$ is the magnetic flux (normalized to $\Phi_{0}$ ) coupled to the target qubit from the microwave and is given by

$$
x_{\varepsilon}(t)=x_{\varepsilon 0} f(t) \sin \omega t,
$$

here, $x_{\varepsilon 0}, f(t)$ and $\omega$ are the peak flux, pulse shape function and frequency of the microwave pulse, respectively. In the case of resonance one has $\omega=\Delta E_{1} / \hbar$.
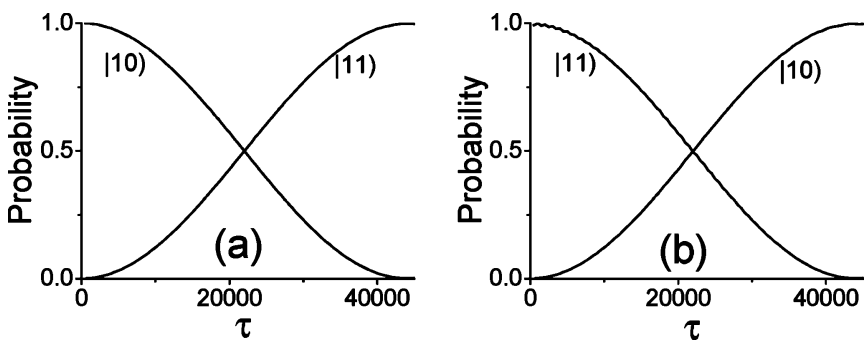

Fig. 5. Evolution of probabilities on states $\mid 10$ ) and |11) of the coupled qubits in a resonant microwave field. The microwave is a square pulse with peak flux of $x_{\varepsilon 0}=2 \times 10^{-4}$. (a) The initial state is $\mid 10$ ) and (b) the initial state is $\mid 11$ ). After the $\pi$-pulse, the coupled qubits flip to the state $\mid 11$ ) in (a) and to the state $\mid 10)$ in (b) and thus the CNOT gate is realized.

Time-dependent wave function $\psi\left(x_{1}, x_{2}, t\right)$ of the coupled qubits is obtained by solving the time-dependent Schrödinger equation

$$
\frac{i \hbar \partial \psi\left(x_{1}, x_{2}, t\right)}{\partial t}=\left[H\left(x_{1}, x_{2}\right)+V\left(x_{1}, x_{2}, t\right)\right] \psi\left(x_{1}, x_{2}, t\right) .
$$

The time-dependent wave function $\psi\left(x_{1}, x_{2}, t\right)$ is expanded in the eigenstate $\mid \alpha$ ) (the eigenenergy is $E_{\alpha}$ ) of $H\left(x_{1}, x_{2}\right)$

$$
\left.\psi\left(x_{1}, x_{2}, t\right)=\sum_{\alpha=1}^{N} c_{\alpha}(t) \mid \alpha\right),
$$

and the expansion coefficients are computed from the matrix equation

$$
\frac{i \partial c_{\alpha}(\tau)}{\partial \tau}=\sum_{\alpha^{\prime}=1}^{N} H_{\alpha \alpha^{\prime}}^{R}(\tau) c_{\alpha^{\prime}}(\tau),
$$

where, $\tau=\omega_{\mathrm{LC}} t$ and $H_{\alpha \alpha^{\prime}}^{R}$ is reduced Hamiltonian matrix element given by

$$
H_{\alpha \alpha^{\prime}}^{R}(\tau)=\frac{\left[E_{\alpha} \delta_{\alpha \alpha^{\prime}}+\left(\alpha\left|V\left(x_{1}, x_{2}, t\right)\right| \alpha^{\prime}\right)\right]}{\hbar \omega_{L C}} .
$$

Using the split-operator method [20] the time-dependent matrix (9) is solved to obtain the expansion coefficients $c_{\alpha}(\tau)$. The probability of being in the state $\mid \alpha)$ is $\left|c_{\alpha}(\tau)\right|^{2}$.

This approach is used to investigate the dynamics of the coupled SQUID flux qubits. The microwave is a rectangular pulse $(f(t)=1)$ with peak flux of $x_{\varepsilon 0}=2 \times 10^{-4}$. In Fig. 5 we plot the evolution of probabilities being in the state |10) and |11) (the probability of being in the other states is almost zero) for the coupled qubits in the resonant microwave field. It is shown that the coupled qubits evolve from the initial state (10) to the final state |11) in (a) and from the initial state |11) to the final state (10) in (b) after the application of a $\pi$-pulse. We also computed the probability for the coupled qubits evolve from the initial states |00) and |01) using the same microwave pulse. It is found that in this case the coupled qubits stayed in the initial states (not shown in this paper). Thus by applying the $\pi$-pulse to the target qubit of the coupled SQUID flux qubits the CNOT gate is realized successfully. 

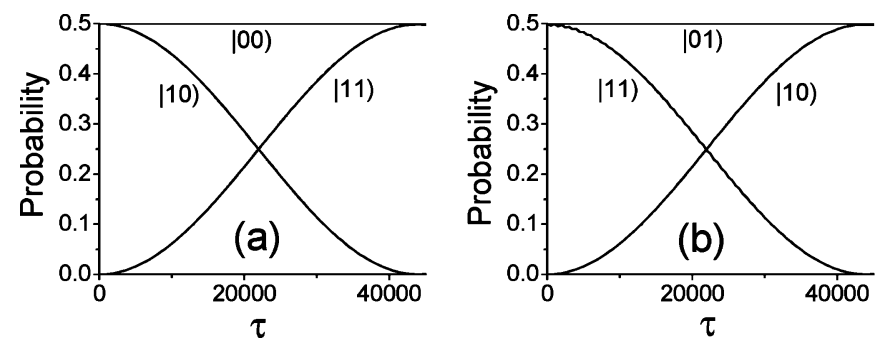

Fig. 6. Evolution of probabilities on states $\mid 10), \mid 00), \mid 11)$, and $\mid 01$ ) of the coupled qubits in a resonant microwave field which is the same as that used in Fig. 5. (a) The initial state is the product state $\mid 10)+\mid 00)$ and (b) the initial state is the product state $\mid 11)+\mid 01)$. After a $\pi$-pulse, the coupled qubits evolve to the entangled state $\mid 00)+\mid 11)$ in (a) and to the entangled state $\mid 01)+\mid 10)$ in (b).

In Fig. 6, we exhibit evolution of the probabilities in states $\mid 10), \mid 00), \mid 11)$, and $\mid 01)$ for the coupled qubits when a microwave pulse the same as that used in Fig. 5 is applied to the target qubit. In this case, the initial state is the product state $\mid 00)+\mid 10)$ in (a) and the product state $\mid 01)+\mid 11)$ in (b). These product states can be created by a $\pi / 2$-pulse applied to the control qubit from the eigenstates $\mid 00)$ and $\mid 01)$, respectively. It is shown that after a $\pi$-pulse is applied to the target qubit the coupled qubits evolve to the entangled state $\mid 00)+\mid 11$ ) in (a) and to the entangled state $\mid 01)+\mid 10$ ) in (b). It is also straightforward to obtain a product state from an entangled state of the coupled qubits with the application of a $\pi$-pulse. Therefore the procedure is reversible.

\section{CONCLUSION}

We proposed a simple method to implement a set of universal two-bit quantum gates using two rf SQUID flux qubits coupled via mutual inductance. We studied the system's dynamics with realistic device parameters and showed that the CNOT gate can be realized successfully by applying the resonant microwave pulse to the target qubit of the coupled qubits. In addition, we demonstrated the use of CNOT to obtain maximally entangled states. This coupling scheme is simple, easy to implement experimentally, and thus promising to extend to many-qubit circuits required for scalable quantum information processing.

\section{REFERENCES}

[1] Y. Makhlin, G. Schon, and A. Shnirman, "Quantum-state engineering with Josephson-junction devices," Rev. Mod. Phys., vol. 73, p. 357, 2001.

[2] Y. Nakamura, Y. A. Pashkin, and J. S. Tsai, "Coherent control of macroscopic quantum states in a single-Cooper-pair box," Nature, vol. 398, p. 786, 1999.

[3] D. Vion et al., "Manipulating the quantum state of an electronic circuit," Science, vol. 296, p. 886, 2002.

[4] Y. Yu, S. Han, X. Chu, S.-I. Chu, and Z. Wang, "Coherent temporal oscillations of macroscopic quantum states in a Josephson junction," Science, vol. 296, p. 889, 2002.

[5] S. Han, Y. Yu, X. Chu, S.-I. Chu, and Z. Wang, "Time-resolved measurement of dissipation-induced decoherence in a Josephson junction," Science, vol. 293, p. 1457, 2001.

[6] J. M. Martinis, S. Nam, J. Aumentado, and C. Urbina, "Rabi oscillations in a large Josephson-junction qubit," Phys. Rev. Lett., vol. 89, p. 117 901, 2002.

[7] J. R. Friedman, V. Patel, W. Chen, S. K. Tolpygo, and J. E. Lukens, "Quantum superposition of distinct macroscopic states," Nature, vol. 406, p. 43, 2000.

[8] C. H. van der Wal et al., "Quantum superposition of macroscopic persistent-current states," Science, vol. 290, p. 773, 2000.

[9] I. Chiorescu, Y. Nakamura, C. J. P. M. Harmans, and J. E. Mooij, "Coherent quantum dynamics of a superconducting flux qubit," Science, vol. 299, p. 1869, 2003.

[10] M. A. Nielsen and I. L. Chuang, Quantum Computation and Quantum Information. Cambridge: Cambridge Univ. Press, 2000.

[11] Y. A. Pashkin et al., "Quantum oscillations in two coupled charge qubits," Nature, vol. 421, p. 823, 2003.

[12] T. Yamamoto, Y. A. Pashkin, O. Astafiev, Y. Nakamura, and J. S. Tsai, "Demonstration of conditional gate operation using superconducting charge qubits," Nature, vol. 425, p. 941, 2003.

[13] A. J. Berkley et al., "Entangled macroscopic quantum states in two superconducting qubits," Science, vol. 300, p. 1548, 2003.

[14] J. E. Mooij et al., "Josephson persistent-current qubit," Science, vol. 285, p. 1036, 1999.

[15] Z. Zhou, S.-I. Chu, and S. Han, "Suppression of energy-relaxation-induced decoherence in a $\Lambda$-type three-level SQUID flux qubits: a darkstate approach," Phys. Rev. B, vol. 70, p. 2004.

[16] Y. Makhlin, G. Schön, and A. Shnirman, "Josephson-junction qubits with controlled couplings," Nature, vol. 398, p. 305, 1999.

[17] V. V. Danilov, K. Likharev, and A. B. Zorin, "Quantum noise in SQUIDs," IEEE Trans. Magn., vol. MAG-19, p. 572, 1983.

[18] Z. Zhou, S.-I. Chu, and S. Han, "Quantum computing with superconducting devices: a three-level SQUID qubit," Phys. Rev. B, vol. 66, p. $054527,2002$.

[19] S.-I. Chu, "The complex-scaling Fourier-grid Hamiltonian method for resonance state problems," Chem. Phys. Lett., vol. 167, p. 155, 1990.

[20] M. R. Hermann and J. A. Fleck Jr., "Split-operator spectral method for solving the time-dependent Schrödinger equation in spherical coordinates," Phys. Rev. A, vol. 38, p. 6000, 1988. 